\title{
Reconsideration of Body Mass Measurement on the International Space Station and Beyond
}

\author{
K. Shimada ${ }^{1}$, Y. Fujii ${ }^{2}$ \\ ${ }^{1}$ Space Medicine Group, Japan Aerospace Exploration Agency \\ JAXA Houston Office, 100 Cyberonics Blvd. STE 201, Houston, TX 77058-2074 USA \\ ${ }^{2}$ Department of Electronic Engineering, Gunma University, Gunma, JAPAN
}

Keywords: International Space Station, Laser Interferometry, Body Mass

\begin{abstract}
In the current space program, astronaut body mass onboard spacecraft, including the International Space Station (ISS) is measured with spring-mass device. We propose a new device "Space Scale" with laser interferometry acceleration measurement and an inline force sensor with a bungee cord. This paper describes concept of Space Scale and its accuracy estimation by ground model test. Accuracy obtained in this ground test was $-0.293 \%$, of which force sensor contributed most to error.
\end{abstract}

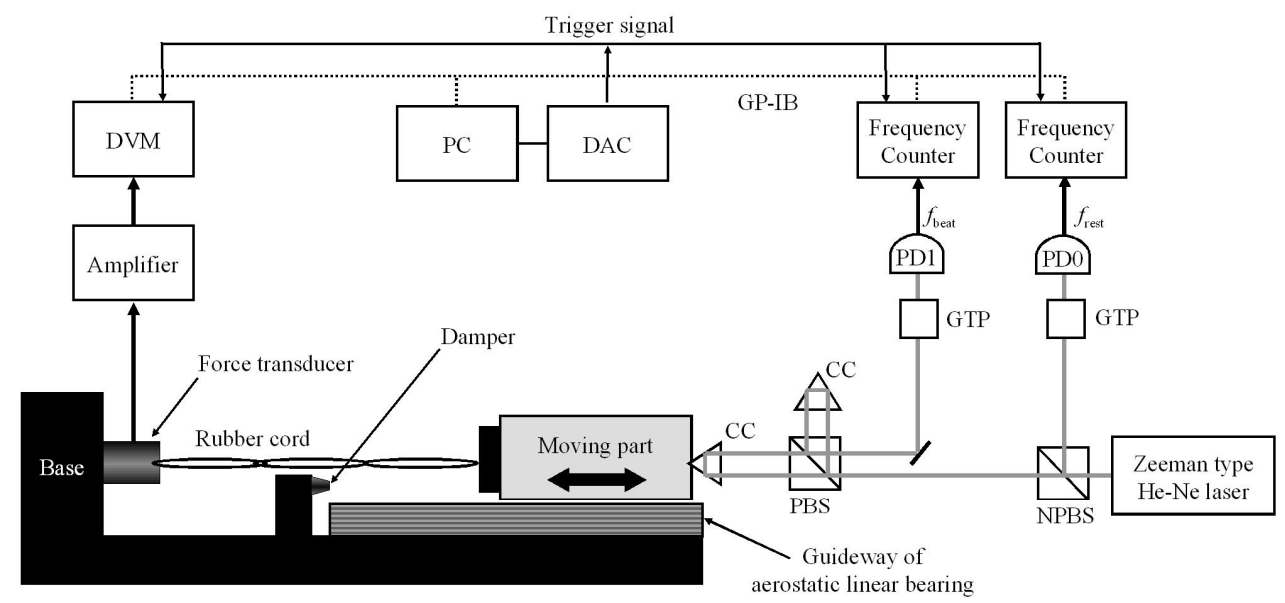

Figure 1. Experimental setup. Code: $\mathrm{CC}=$ cube corner prism, $\mathrm{PBS}=$ polarizing beam splitter, NPBS= non-polarizing beam splitter, GTP $=$ Glan-Thompson prism, $\mathrm{PD}=$ photo diode, $\mathrm{DAC}=$ digital-toanalog converter, $\mathrm{DVM}=$ digital volt meter, $\mathrm{PC}=$ computer.

\section{Introduction}

The body mass is the basic index of nourishment, as well as altered body fluid homeostasis under microgravity environment in the spacecraft in low earth orbit. Without periodic onboard medical monitoring and assessment by flight surgeons on the ground, astronauts tend to be too hypovolemic (low in body fluid). Hypovolemia at the time of reentry back to the ground enhances physiologic intolerance including low blood pressure, which might lead to dysfunction of returning crew at emergency landing.

Measuring astronaut body mass on a spacecraft in low earth orbit on a regular basis is now a part of space medical operations on the International Space Station (ISS). However, its measurement principle in the microgravity environment has not changed much since the Skylab program in 1970's. 
Skylab body mass measurement device was based on mass-spring system resonant frequency and developed by Thornton WE et al. at NASA.

The apparatus for in-orbit body mass measurement started as payload experiment hardware. Initially, size and volume of the hardware and complexity of operation was not a big issue. When it became part of the periodical systems operation, simplicity of protocol and easiness of hardware manipulation is now more important to save precious crew time on the spacecraft.

Apparently in microgravity, ground-type balance or scale is inapplicable. In the previous space programs, selected quality spring with timer device was chosen to implement a spring-mass system and frequency principle design to derive the amount of mass. Other measurement principles were hard to materialize. In the current ISS program, NASA ISS "SLAMMD" body mass measurement device uses spring-mass system with specially made spring. Instead of timer, acceleration sensor is used to calculate mass. Still SLAMMD needs a rigid frame to oscillate whole human body. It is installed in a standard ISS experiment rack and not easy to deploy, and needs considerable crew time to relocate.

Now it is the time to think about applying modern laser technique to observe distance, velocity, and use force sensors to this old problem of measurement of mass in microgravity. This paper proposes a new look at this old problem with the current optics technology. We named our device "Space Scale" (Fujii).

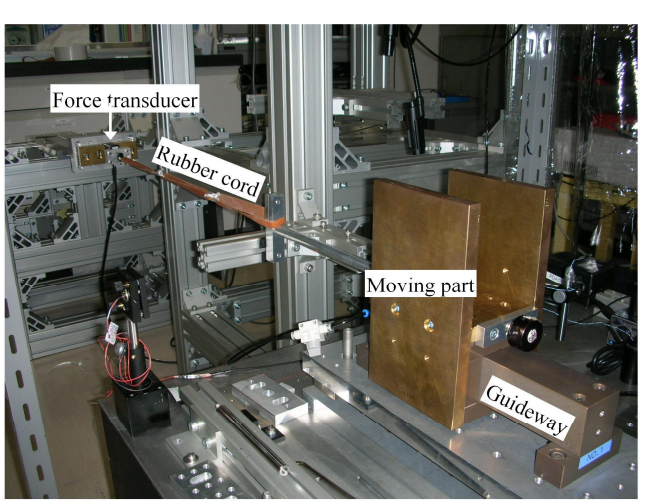

Figure 2. Photograph of the experimental setup.

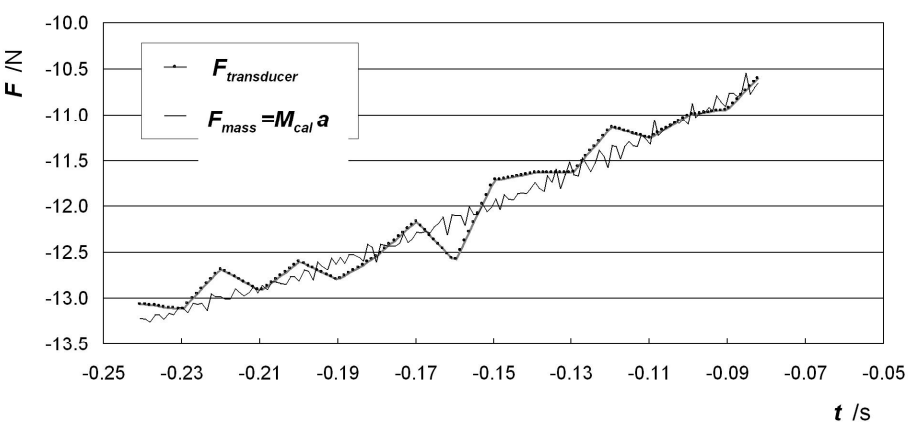

Figure 3. Force measured using force transducer and optical interferometer

\section{Requirements}

Body mass measurement on ISS is done every month for every astronaut onboard. ISS medical requirement is the base of this periodic body mass measurement. Current baseline instrument is a Russian IN Body Mass Measurement Device in the Service Module of the Russian segment of the ISS. Its design principle is based on resonant frequency of mass-spring system. The Russian device is semi-permanently installed onto the Russian module floor of the ISS. NASA activated a rack-mounted device called SLAMMD in 2006, which measures acceleration of mass in a mass-spring system. These two need a relatively large frame to accommodate a human body, which leads to a permanent installation. From operational view, it is desired that the device to be much smaller and transportable to eliminate mechanical interference with other apparatus in a module, and save crew time to deploy the device. Thus the first condition for our new device is: (1) Compactness and portability.

A mass measurement requires reasonable precision. In case of human body, required precision is the same as body weight measurement on the ground for medical purpose. Clinical-use devices have precision about $100 \mathrm{~g}$, or $0.13-0.2 \%$ (weight of the subject centers around $75 \mathrm{~kg}$ ). To discuss average 
weight changes over a number of subjects, precision around this range is required: (2) Precision of $0.2 \%$ over body mass range $(\sim 100 \mathrm{~kg})$.

It is ideal to achieve the precision of $0.05 \mathrm{~kg}$ to be reliable enough to discuss body mass change in the orbit. Accuracy requirement depends on how easy it is to calibrate. Because the measurement is done over a brief time, long-term drift is not an issue, if some means of calibration is available. Practically this is achieved by measuring a calibration standard mass, which is available in the form of metal hardware of known mass in the cabin.

\section{Ground Model Study}

Based on "Space Scale" principle (Fujii and Shimada, 2006), prototype ground model was submitted for precision estimation. This design incorporates an attachment of an elastic spring to the vicinity of human body center of gravity, laser interferometry distance measurement that makes it a compact device, and a force sensor to record the tension of the elastic spring. The data showed that its precision generally covers the requirements described above.

\section{Methods}

Desk-top bread board model was constructed with simulated subject mass and rubber cord (Fig.1, 2). Microgravity is simulated by use of aerostatic linear bearing. This setup was made for proof-of-concept and estimation of accuracy/precision. Simulated body mass $M_{\mathrm{cal}}$ and rubber property values were arbitrary, but large enough to model human body mass measurement. To calculate the value of mass, the acceleration of simulated body when rubber cord was stretched and released was measured by laser interferometry, and the force was recorded by a transducer as rubber cord tension. The relationship of the body acceleration and force between the ISS wall and the astronaut subject gives the value of mass. Details of hardware and measurement description were previously reported (Fujii).

Orbit model configuration is designed as Fig.4. The absolute reference is the ISS structure. The benefit of this design is that no large frame nor long bar needs to be deployed. Precision is expected from the use of large ISS module space, which enables the subject astronaut to travel a relatively long distance on the laser beam line. To improve handling easiness, active components would reside at the wall side of the system. Bungee cord is a standard item for exercise on ISS, so it is convenient in regard to re-supply.

\section{Results}

The ground model worked and produced mass values as designed. Accuracy was assessed with a simulated body mass of $M_{\text {cal }}=11.628 \mathrm{~kg}$. The spring constant of the bungee cord produced body acceleration of around $1 \mathrm{~ms}^{-2}$. The force value sensed by the transducer is shown in Fig. 3, together with derived measurement value of $M_{\text {meas. }}$. Mean of $M_{\text {meas }}$ was $11.5937 \mathrm{~kg}$, which was $0.293 \%$ smaller than the true value.

\section{Discussion}

The accuracy of the measurement result of $M_{\text {meas }}$ was promising for medical use, but not as good as expected. Distance and acceleration measurement by laser interferometry worked as in previous reports (Fujii), as verified by smooth data trend plotted against body displacement. The force values in Fig. 3 show fluctuation. The force sensor output curve against time in Fig. 3 was not smooth. It eventually resulted in compromised precision of $M_{\text {meas }}$.

It was concluded that performance of the force sensor was not optimal, and it was the reason why the result accuracy was worse than expected. Selection of the force sensor and its mechanical 
implementation would be the key for Space Scale. Reducing bungee cord spring constant, to use lower acceleration might increase accuracy and precision.

The elasticity and subject reflex in changing body shape affects the accuracy and precision of the body mass measurement. Our prediction is that training would eliminate these factors. This aspect should be tested aboard parabolic flight aircraft.

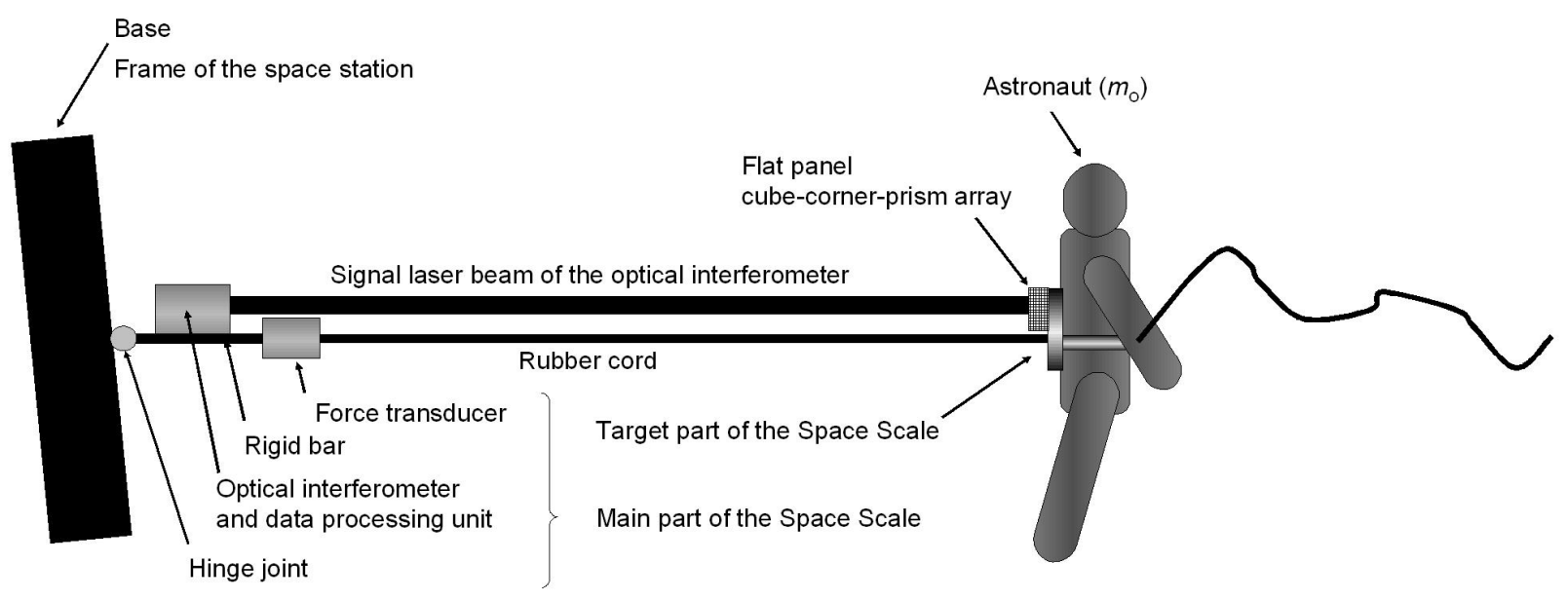

Figure 4. Space Balance for Astronaut Mass Measurement

1

\section{Conclusion and Future Study}

Working with the ground model it was concluded that flight model could be a small-volume device that suffices ISS and future Mars mission medical operations requirements. We are to test fly a second ground prototype on a parabolic flight to evaluate its operability. The same device would be suitable for measuring payload mass onboard ISS and other spacecraft for logistics purpose. If the device is tuned for smaller mass, it should be able to support onboard experiment sample assessment.

\section{Acknowledgments}

This study was supported by a research-aid fund of the Japan Space Forum.

\section{References}

[1] W. Thornton and J. Ord: Specimen mass measurement, NASA Tech. Report, N74-11867, 1974.

[2] D.C. Smith and K.A. Kaufman: Space Linear Acceleration Mass Measurement Device (SLAMMD) for the Human Research Facility (HRF), SAE 981652: 1-23, 1998.

[3] Y. Fujii and K. Shimada, "Space Scale: Instrument for Astronaut Mass Measurement", Proc. ISTS 2006, No.2006-p-01 (Kanazawa, Japan) June 2006.

[4] Y. Fujii, "Measurement of impulse response of force transducers", Rev. Sci. Instrum., Vol.72, No.7, pp. 3108-3111, 2001. 